\title{
SECOND ORDER APPROXIMATION FOR OPTICAL POLARONS IN THE STRONG COUPLING CASE
}

\author{
N.N.Bogolubov, JR. \\ V.A.Steklov Mathematical Institute, Russian Academy of Sciences \\ GSP-1, 117966 Moscow, Russia
}

Received September 12, 1995

\begin{abstract}
Here we propose a method of construction of the second order approximation of the ground state energy for a class of model Hamiltonians with linear type interaction with respect to Bose operators in the strong coupling case. For the application of the above-mentioned method we have considered polaron model and proposed a set of nonlinear differential equations for the ground state energy calculation in the strong coupling case. We have considered also the radially symmetrical case.
\end{abstract}

\section{Introduction}

Here we shall construct the second order approximation for problem of optical polaron in the strong coupling case. Finally we shall construct from this theory a set of nonlinear differential equations which solution can be obtained by numerical calculation. We specially consider also spherically symmetric case.

Polaron theory is the simplest application of quantum field theory to solid state physics but it nevertheless reflects many typical properties of quantum particle interacting with quantum field. Therefore the results derived from the polaron theory are of interest for many related regions of physics. As an example one can note the theory of mobility of ions injected into liquid helium.

The question of the possible existence of polaron states distinct from the ground state is basic as to the study of processes related to excitations in polar media, namely photo excitation of $F$-centers and other lattice defects [1].

At present the problem of excited polaron states besides being of theoretical interest is gaining attention because of the problem of electron transfer excitations in a broad variety of condensed media (solutions of biomacromoleculers, organic semiconductors, etc. [2]).

Considering moving particle interacting with quantum field it is usual to consider the Hamiltonian of the type $H=H_{s}+H_{\Sigma}+H_{\text {int }}$, where $H_{s}$ is the energy of the particle, $H_{\Sigma}$ - the energy of quantum field and $H_{\text {int }}$ - the interaction energy. As usually in order to deal with discrete spectrum only the whole system is considered in some finite space $V$ (volume) for instance in a cube $V=L^{3}$ and some periodical boundary conditions are imposed. 
Of course one always has in mind limiting passage $V \rightarrow \infty$ leading to continuous spectrum.

If considered quantum field can be represented as a set of noninteracting oscillators, then

$$
H_{\Sigma}=\frac{1}{2} \sum_{(f)} \hbar \omega_{f}\left(b_{f} b_{f}^{+}+b_{f}^{+} b_{f}\right)
$$

where $\omega_{f}$ are frequencies of oscillators and $b_{f}^{+}, b_{f}$ are quantum amplitudes with usual commutation relations which correspond to Bose-statistics.

Often the interaction energy is represented by the expression which is spatially uniform and linear dependent on a quantum field function, for example

$$
H_{i n t}=\int \mathrm{d} \boldsymbol{r}^{\prime} K\left(\boldsymbol{r}-\boldsymbol{r}^{\prime}\right) \Psi\left(\boldsymbol{r}^{\prime}\right)+\int \mathrm{d} \boldsymbol{r}^{\prime} K^{*}\left(\boldsymbol{r}-\boldsymbol{r}^{\prime}\right) \Psi^{+}\left(\boldsymbol{r}^{\prime}\right) .
$$

Expanding $\Psi(\boldsymbol{r})$ as usually adopted in the secondary quantization formalism

$$
\Psi(\boldsymbol{r})=\sum_{(f)} b_{f} \frac{e^{\mathrm{i}(\boldsymbol{f} \boldsymbol{r})}}{\sqrt{V}}
$$

we get the following expression

$$
H_{i n t}=\sum_{(f)}\left\{U_{f} e^{\mathrm{i}(\boldsymbol{f} \boldsymbol{r})} b_{f}+U_{f}^{*} e^{-\mathrm{i}(\boldsymbol{f} \boldsymbol{r})} b_{f}^{+}\right\}
$$

in which magnitude $U_{f}, U_{f}^{*}$ are proportional to $1 / \sqrt{V}$.

Here we use typical expressions for $H_{s}, H_{\Sigma}, H_{\text {int }}$. So we can write our Hamiltonian in the form

$$
H=\frac{\boldsymbol{P}^{2}}{2 m}+\frac{1}{2} \sum_{(f)} \hbar \omega_{f}\left(b_{f} b_{f}^{+}+b_{f}^{+} b_{f}\right)+\sum_{(f)}\left\{U_{f} e^{\mathrm{i}(\boldsymbol{f} \boldsymbol{r})} b_{f}+U_{f}^{*} e^{-\mathrm{i}(\boldsymbol{f} \boldsymbol{r})} b_{f}^{+}\right\} .
$$

This Hamiltonian is considered in many different physical applications (for historical reasons mainly). It is possible to mention for instance about a problem of moving particle of admixture in helium II [3-5] or about moving electron in semiconductor $[6,7]$ or about interaction of a nucleon with scalar meson field in the nonrelativistic approximation. Hamiltonians of more complicated form are investigated also in nonrelativistic theories of interaction of electron with electromagnetic field, nucleon with pseudoscalar and vector meson field and interaction of radiation with matter [8-10].

We notice that although such a Hamiltonian is one of the simplest in the problems of interaction of a particle with a quantum field the exact solution to the correspondent wave equation is impossible, therefore it is necessary to use approximate methods of perturbation series. The scheme of perturbation theory for the weak interaction case when $H_{i n t}$ is a small perturbation term and $H_{s}, H_{\Sigma}$ are the main terms is well known. However in some problems the field-particle coupling is not small. One can point to some cases of strong coupling when $H_{\text {int }}$ is proportional to a large parameter as well as to mathematically similar case of "adiabatic coupling" when the small term is the kinetic energy of the field as it is in the case of electron moving in ion crystal $[6,7]$. 
We notice that some problems where kinetic energy can be considered as a small perturbation term have been considered by means of perturbation method. We can mention the problem nucleus movement influence on energy levels of electron spectrum in metal [11] for example. However in all these resolved problems the absence of translational degeneracy is essential. In our case of particle-field interaction translational degeneracy always exists because the Hamiltonian (1) is invariant with respect to translational group

$$
\boldsymbol{r} \rightarrow \boldsymbol{r}+\boldsymbol{\alpha}, \quad \boldsymbol{\alpha}=\mathrm{const}, \quad b_{f} \rightarrow b_{f} e^{-\mathrm{i}(\boldsymbol{f} \boldsymbol{r})}, \quad b_{f}^{+} \rightarrow b_{f}^{+} e^{\mathrm{i}(\boldsymbol{f} \boldsymbol{r})} .
$$

Because of this degeneracy the well-known method of adiabatic approximation turns out to be not accessible here and so it is necessary to construct new special (peculiar) form of the perturbation theory. A new scheme of perturbation theory was elaborated by N.N.Bogolubov [12]. Recently many interesting results were obtained by Lakhno and co-workers also within frames of adiabatic theory in the case of a polar medium when the particle-field strong coupling approximation can be applied [14]. For spherically symmetric case they have constructed a system of nonlinear differential equations and also got in piezoelectric medium a nonlinear Schrödinger equation with a cubic nonlinearity like

$$
y^{\prime \prime}+\frac{2}{x} y^{\prime}+y^{3}-y=0, \quad y^{\prime}(0)=y(\infty)=0
$$

This equation has been analysed in [13].

Here in this article we are going to construct the second order approximation for problems of optical polaron in the strong coupling case. We start with some ideas given by N.N.Bogolubov in [14].

\section{Approximation by variational principle. Polaron at rest.}

In this article we shall consider the Hamiltonian

$$
H=\frac{P^{2}}{2 m}+\frac{1}{\sqrt{V}} \sum_{(f)} U_{f}\left(b_{f} e^{\mathrm{i} f x}+b_{f}^{+} e^{-\mathrm{i} f x}\right)+\sum_{(f)} \omega_{f} b_{f}^{+} b_{f} .
$$

Here

$$
f=\boldsymbol{f}=\left(\frac{2 \pi n_{1}}{L}, \frac{2 \pi n_{2}}{L}, \frac{2 \pi n_{3}}{L}\right),
$$

$n$ - integers, $L^{3}=V, x=r, P=-\mathrm{i} \frac{\partial}{\partial x}, \omega_{f}$ and $U_{f}$ are supposed to be radially symmetric with respect to $f: U_{f}=U_{|f|}, \omega_{f}=\omega_{|f|} ; b_{f}, b_{f}^{+}$- Bose amplitudes.

State vector $\Phi$ corresponding to the lowest energy is defined by

$$
\left\langle\Phi^{*} H \Phi\right\rangle=\min =\varepsilon, \quad\left\langle\Phi^{*} \Phi\right\rangle=1 .
$$

To obtain an approximation we shall now construct the trial state vectors to insert them into relation (2). Put

$$
b_{f}=c_{f}+a_{f}, \quad b_{f}^{+}=c_{f}^{*}+a_{f}^{+},
$$


where $c_{f}, c_{f}^{*}$ are $c$-numbers and $a_{f}, a_{f}^{+}$- Bose amplitudes. Let's introduce vacuum state vector $\Phi_{v}$ for $a_{f}, a_{f}^{+}$operators:

$$
\left\langle\Phi_{v}^{*} \Phi_{v}\right\rangle=1, \quad a_{f} \Phi_{v}=0, \quad \Phi_{v}^{*} a_{f}^{+}=0
$$

and define trial state vector $\Phi$ :

$$
\Phi=\varphi(x) \Phi_{v}, \quad \int \mathrm{d} x \varphi^{*}(x) \varphi(x)=1 .
$$

Here function $\varphi(x)$ and $c$-numbers $c_{f}$ are to be determined by the variational principle:

$$
\Lambda\left(\varphi ; \ldots c_{f} \ldots\right)=\min ,
$$

where

$$
\Lambda\left(\varphi ; \ldots c_{f} \ldots\right)=\int \mathrm{d} x\left\langle\Phi_{v}^{*} \varphi^{*}(x) H \varphi(x) \Phi_{v}\right\rangle
$$

and therefore

$$
\Lambda=\int \mathrm{d} x \varphi^{*}(x)\left\{\frac{P^{2}}{2 m}+\frac{1}{\sqrt{V}} \sum_{(f)} U_{f}\left(c_{f} e^{\mathrm{i} f x}+c^{*} e^{-\mathrm{i} f x}\right)+\sum_{(f)} \omega_{f} c_{f}^{*} c_{f}\right\} \varphi(x) .
$$

Because of the minimal property of $\Lambda$ with respect to $c_{f}$ we have

$$
\frac{\partial \Lambda}{\partial c_{f}}=0, \quad \frac{\partial \Lambda}{\partial c_{f}^{*}}=0
$$

and therefore

$$
\begin{gathered}
c_{f}^{*}=-\frac{U_{f}}{\omega_{f} \sqrt{V}} \int \mathrm{d} x e^{\mathrm{i} f x} \varphi^{*}(x) \varphi(x)=c_{f}^{*}(\varphi), \\
c_{f}=-\frac{U_{f}^{*}}{\omega_{f} \sqrt{V}} \int \mathrm{d} x e^{-\mathrm{i} f x} \varphi^{*}(x) \varphi(x)=c_{f}(\varphi) .
\end{gathered}
$$

We see from equation (7) that the expression

$$
\Lambda(\varphi)=\Lambda\left(\varphi ; \ldots c_{f}(\varphi) \ldots c_{f}^{*}(\varphi) \ldots\right)
$$

must have the minimal property with respect to $\varphi(x)$ and thus:

$$
\delta_{\varphi} \Lambda(\varphi)=0
$$

So we have

$$
\Lambda(\varphi)=\int \mathrm{d} x \varphi^{*}(x)\left\{\frac{P^{2}}{2 m}+\mathrm{U}(x ; \varphi)\right\} \varphi(x),
$$

where

$$
\mathrm{U}(x ; \varphi)=\frac{1}{\sqrt{V}} \sum_{(f)} U_{f}\left(c_{f}(\varphi) e^{\mathrm{i} f x}+c^{*}(\varphi) e^{-\mathrm{i} f x}\right)+\sum_{(f)} \omega_{f} c_{f}^{+}(\varphi) c_{f}(\varphi) .
$$


From equation (8) it follows that:

$$
\int \mathrm{d} x \varphi^{*}(x)\{\delta \mathrm{U}(x ; \varphi)\} \varphi(x)=0
$$

and we have from equation (9)

$$
\begin{aligned}
\delta_{\varphi} \Lambda(\varphi)= & \int \mathrm{d} x\left(\delta \varphi^{*}(x)\right)\left\{\frac{P^{2}}{2 m}+\mathrm{U}(x ; \varphi)\right\} \varphi(x)+ \\
& \left.\int \mathrm{d} x \varphi^{*}(x)\right)\left\{\frac{P^{2}}{2 m}+\mathrm{U}(x ; \varphi)\right\}(\delta \varphi(x))=0 .
\end{aligned}
$$

Here

$$
\int \mathrm{d} x\left(\delta \varphi^{*}(x)\right) \varphi(x)+\int \mathrm{d} x \varphi^{*}(x)(\delta \varphi(x))=0 .
$$

Therefore:

$$
\left\{\frac{P^{2}}{2 m}+\mathrm{U}(x ; \varphi)\right\} \varphi(x)=E \varphi(x) .
$$

As it follows from equation (10) that $E=\Lambda(\varphi)$. Hence in order to satisfy the minimal principle $\Lambda(\varphi)=$ min we must find the solution to equation (12) for which $E=E_{0}$ is minimal. Because of our particular choice for the trial state vectors $(6)$ it is clear that $E_{0} \geq \mathcal{E}$.

It is to be noted that the equation (12) had been obtained at one time by Landau and Pekar in a different way as well as the expression for the mass of a moving polaron. In the paper [12] engaging the adiabatic approximation these results were derived as a first approximation. Remark also that

$$
\begin{aligned}
E_{0} & =\min \Lambda(\varphi)=\min _{\varphi} \min _{c} \Lambda\left(\varphi ; \ldots c_{f} \ldots\right) \leq \min _{\varphi} \Lambda(\varphi ; \ldots 0 \ldots) \\
& =\min _{\varphi} \int \mathrm{d} x \varphi^{*}(x) \frac{P^{2}}{2 m} \varphi(x)=0,
\end{aligned}
$$

thus $\mathcal{E} \leq E_{0} \leq 0$.

Suppose now that the equation (12) has such a solution $\varphi=\varphi_{0}(x)$, $E=E_{0}$ so that on associated linear equation

$$
\left\{\frac{P^{2}}{2 m}+\mathrm{U}\left(x, \varphi_{0}\right)\right\} \varphi(x)=E \varphi(x)
$$

has for $E=E_{0}$ the unique (apart from the arbitrary constant multiplier) solution $\varphi=\varphi_{0}$. So for all other eigenvalues of equation (14) one has $E_{n}>E_{0}$. Since from equation (11) it follows that $\mathrm{U}^{*}(x, \varphi)=\mathrm{U}(x, \varphi)$ we can take $\varphi_{0}$ to be real. Let's further remark that

$$
\begin{aligned}
\mathrm{U}(x, \varphi)= & -\frac{2}{V} \sum_{(f)} \frac{U_{f}^{2}}{\omega_{f}}\left\{\int \mathrm{d} x e^{-\mathrm{i}(f x)} \varphi^{*}(x) \varphi(x)\right\} e^{\mathrm{i}(f x)} \\
& +\frac{1}{V} \sum_{(f)} \frac{U_{f}^{2}}{\omega_{f}}\left|\int \mathrm{d} x e^{-\mathrm{i}(f x)} \varphi^{*}(x) \varphi(x)\right|^{2}
\end{aligned}
$$


and hence

$$
\begin{aligned}
\mathrm{U}(x, \varphi)= & -\int \mathrm{d} y K(x-y) \varphi^{*}(y) \varphi(y) \\
& +\frac{1}{2} \int \mathrm{d} x \mathrm{~d} y K(x-y) \varphi^{*}(x) \varphi(x) \varphi^{*}(y) \varphi(y),
\end{aligned}
$$

where

$$
K(x)=\frac{2}{V} \sum_{(f)} \frac{U_{f}^{2}}{\omega_{f}} e^{\mathrm{i}(f x)}=\frac{2}{(2 \pi)^{3}} \int \mathrm{d} f \frac{U_{f}^{2}}{\omega_{f}} e^{\mathrm{i}(f x)} .
$$

Since $U_{f}^{2} / \omega_{f}$ is radially symmetrical with respect to $f$ the expression is a radially symmetrical function of $x: K(x)=K(|x|)$. Therefore if $\varphi(x)$ is radially symmetrical, then $\mathrm{U}(x, \varphi)$ also possesses this property. Thus in the considered situation the operator

$$
\frac{P^{2}}{2 m}+\mathrm{U}(x, \varphi)
$$

conserves the property of radial symmetry. Hence we may consider the radially symmetrical solutions to equation (12). We suppose that one of them, $\varphi_{0}(x)$, corresponds to the lowest possible value of energy $E_{0}$. It is to be stressed that if $\varphi(x)$ is a solution to the equation (12) then $\varphi(x+\zeta)$ will also satisfy this equation for arbitrary constant $\zeta$. Such translation may be considered as a translation of coordinate system in $(x)$-space by fixing the zero-point anywhere in space. We thus see that we are in the degeneracy situation and so our averages computed by means of $\varphi_{0}(x)$ are what is called the quasiaverages. Let's fix somehow the zero point origin of the coordinate system, then $\varphi_{0}(x)=f(|x|)$ is completely determined and so $\mathrm{U}\left(x, \varphi_{0}\right)=\mathrm{U}(|x|)$. Therefore the linear equation (14)

$$
\left\{\frac{P^{2}}{2 m}+\mathrm{U}(|x|)\right\} \varphi(x)=E \varphi(x)
$$

is no more translationally invariant and we may suppose that its solution $\Psi(x)=\varphi_{0}(x)=f(|x|)$ for $E=E_{0}$ is unique, apart from the arbitrary constant multiplier for $\Psi(x)$.

Then we consider the adiabatic approximation. We choose here that

$$
U_{f}=\frac{\varepsilon}{\sqrt{2}} A_{f}, \quad \omega_{f}=\varepsilon^{2} \nu_{f}
$$

where $\varepsilon$ is a small parameter. Our Hamiltonian (2) takes the form

$$
H=\frac{P^{2}}{2 m}+\frac{\varepsilon}{V} \sum_{(f)} \frac{A_{f}}{\sqrt{2}}\left(b_{f} e^{\mathrm{i} f x}+b_{f}^{+} e^{-\mathrm{i} f x}\right)+\varepsilon^{2} \sum_{(f)} \nu_{f} b_{f}^{+} b_{f} .
$$

Let's introduce like previously in equation (4) new Bose amplitudes

$$
b_{f}=c_{f}+a_{f}, \quad c_{f}=\frac{U_{f}}{\varepsilon}, \quad U_{f}=-\frac{A_{f}}{\sqrt{2 V} \nu_{f}} \int \mathrm{d} x e^{-\mathrm{i}(f x)} \varphi_{0}^{2}(x),
$$


where $\varphi_{0}(x)$ is the same function as in equation (13). Then

$$
H=\Gamma+\varepsilon\left\{\frac{1}{\sqrt{2 V}} \sum_{(f)} A_{f}\left(a_{f} e^{\mathrm{i} f x}+a_{f}^{+} e^{-\mathrm{i} f x}\right)\right\}+\varepsilon^{2} \sum_{(f)} \nu_{f} a_{f}^{+} a_{f},
$$

where

$$
\Gamma=\frac{P^{2}}{2 m}+\mathrm{U}\left(x, \varphi_{0}\right)
$$

The first order approximation Hamiltonian may be obtained by dropping the terms of the order $\varepsilon, \varepsilon^{2}$. In this approximation we have the same equation which was obtained in equation (3) by using the variational principle. Here $\Phi=\varphi_{0}(x) \Phi_{v}$. Because $a_{f}^{+} a_{f} \Phi_{v}=0$ we see that we also may take as the first order approximation for the Hamiltonian the following expression:

$$
H_{0}=\Gamma+\varepsilon^{2} \sum_{(f)} \nu_{f} a_{f}^{+} a_{f}=\Gamma+\sum_{(f)} \omega_{f} a_{f}^{+} a_{f} .
$$

The inclusion of the $\varepsilon^{2}$ order term into this Hamiltonian clearly does not change the result

$$
\Phi=\varphi_{0}(x) \Phi_{v}, \quad H_{0} \varphi_{0}(x) \Phi_{v}=E_{0} \varphi_{0}(x) \Phi_{v} .
$$

Let us consider the second approximation. Let's write

$$
\begin{aligned}
& H=H_{0}+\varepsilon H_{1} \\
& H_{0}=\Gamma+\sum_{(f)} \omega_{f} a_{f}^{+} a_{f} \\
& H_{1}=\sum_{(f)} \nu_{f}\left(U_{f}^{*} a_{f}+U_{f} a_{f}^{+}\right)+\frac{1}{\sqrt{V}} \sum_{(f)} \frac{A_{f}}{\sqrt{2}}\left(a_{f} e^{\mathrm{i} f x}+a_{f}^{+} e^{-\mathrm{i} f x}\right)
\end{aligned}
$$

and insert into the equation for the state vector $H \Phi=E \Phi$ the expansion

$$
\begin{aligned}
& \Phi=\Phi_{0}+\varepsilon \Phi_{1}+\varepsilon^{2} \Phi_{2}+\ldots, \\
& \Phi_{0}=\varphi_{0}(x) \Phi_{v} \\
& E=E_{0}+\varepsilon \Delta_{1}+\varepsilon^{2} \Delta_{2}+\ldots
\end{aligned}
$$

Therefore

$$
\begin{gathered}
\left(H_{0}+\varepsilon H_{1}\right)\left(\Phi_{0}+\varepsilon \Phi_{1}+\varepsilon^{2} \Phi_{2}+\ldots\right)= \\
\left(E_{0}+\varepsilon \Delta_{1}+\varepsilon^{2} \Delta_{2}+\ldots\right)\left(\Phi_{0}+\varepsilon \Phi_{1}+\varepsilon^{2} \Phi_{2}+\ldots\right)
\end{gathered}
$$

To satisfy this equation we take

$$
\begin{aligned}
& \left(H_{0}-E_{0}\right) \Phi_{0}=0, \\
& \left(H_{0}-E_{0}\right) \Phi_{1}=\Delta_{1} \Phi_{0}-H_{1} \Phi_{0}, \\
& \left(H_{0}-E_{0}\right) \Phi_{2}=\Delta_{2} \Phi_{0}+\Delta_{1} \Phi_{1}-H_{1} \Phi_{1} .
\end{aligned}
$$

Let us observe that by virtue of equations (4), (10)

$$
\int \mathrm{d} x \varphi_{0}(x) H_{1} \varphi_{0}(x)=0 .
$$


Hence $\left\langle\Phi_{0}^{*} H_{1} \Phi_{0}\right\rangle=0$ or

$$
\left\langle\Phi_{0}^{*}\left(H_{0}-E_{0}\right) \Phi_{0}\right\rangle=\Delta_{1}-\left\langle\Phi_{0} H_{1} \Phi_{0}\right\rangle=0 .
$$

We thus have

$$
\Delta_{1}=0, \quad\left(H_{0}-E_{0}\right) \Phi_{0}=-H_{1} \Phi_{0},
$$

that is:

$$
\begin{gathered}
\left(\Gamma-E_{0}+\sum_{(f)} \omega_{f} a_{f}^{+} a_{f}\right) \Phi_{1}=-H_{1} \Phi_{0}= \\
-\frac{1}{\sqrt{2 V}} \sum_{(f)} A_{f} a_{f} e^{-\mathrm{i} f x} \varphi_{0}(x) \Phi_{v}+\sum_{(f)} \nu_{f} U_{f} a_{f}^{+} \varphi_{0}(x) \Phi_{v} .
\end{gathered}
$$

Let us now expand $\Phi_{1}$ in Fourier series of eigenfunctions

$$
\begin{gathered}
\left(\Gamma-E_{n}\right) \varphi_{n}(x)=0, \quad n \geq 0, \\
\int \mathrm{d} x \varphi_{n}^{*}(x) \varphi_{m}(x)= \begin{cases}1, & n=m, \\
0, & n \neq m .\end{cases}
\end{gathered}
$$

Noting that according to equation (17)

$$
\int \mathrm{d} x \varphi_{0}(x) H_{1} \Phi_{0}=0,
$$

we obtain

$$
\Phi_{1}=-\frac{1}{\sqrt{2 V}} \sum_{n>0} \varphi_{n}(x) \sum_{(f)} \frac{A_{f} \int \mathrm{d} x e^{-\mathrm{i} f x} \varphi_{n}(x) \varphi_{0}(x)}{E_{n}-E_{0}+\omega_{f}} a_{f}^{+} \Phi_{v} .
$$

Further we have $\left(H-E_{0}\right) \Phi_{2}=\Delta_{2} \Phi_{0}-H_{1} \Phi_{1}$. But $\left\langle\Phi_{0}^{*}\left(H_{0}-E_{0}\right) \Phi_{2}\right\rangle=0$ and it follows that $\Delta_{2}=\left\langle\Phi_{0}^{*} H_{1} \Phi_{1}\right\rangle$. Remark now that by virtue of equation (18)

$$
\int \mathrm{d} x \varphi_{1}(x) \sum_{(f)}\left(\nu_{f} U_{f} a_{f}^{+}+\nu_{f} U_{f}^{*} a_{f}\right) \varphi_{n}(x)=0 .
$$

Therefore

$$
\Delta_{2}=-\frac{1}{2 V} \sum_{(f)} \sum_{n>0} \frac{\left|\int \mathrm{d} x e^{-\mathrm{i} f x} \varphi_{n}^{*}(x) \varphi_{0}(x)\right|^{2}}{E_{n}-E_{0}+\omega_{f}} .
$$

\section{Second approximation for optical polaron problem in the strong coupling case}

Being based on the discussed adiabatic approximation for the Hamiltonian (2) we construct the second order approximation for the ground state energy $\mathcal{E}: \mathcal{E}=E_{0}+\varepsilon^{2} \Delta_{2}+O\left(\varepsilon^{4}\right)+\ldots$, where we can rewrite equation (19) as

$$
\Delta_{2}=-\frac{1}{2 V} \sum_{n>0} \sum_{(f)} A_{f}^{2} \int \mathrm{d} x \mathrm{~d} x^{\prime} e^{\mathrm{i} f\left(x-x^{\prime}\right)} \Phi_{0}^{*}(x) \Phi_{0}\left(x^{\prime}\right) \frac{\Phi_{n}^{*}(x) \Phi_{n}\left(x^{\prime}\right)}{E_{n}-E_{0}+\varepsilon^{2} \nu_{f}} .
$$

Here functions $\Phi_{0}, \Phi_{n}$ satisfy the equation

$$
\Gamma \Phi_{n}=E_{n} \Phi_{n}, \quad n \geq 0
$$


and the Hamiltonian $\Gamma$ according to equations (14), (15) has the form

$$
\begin{aligned}
\Gamma= & -\frac{\Delta_{x}}{2 m}-\frac{1}{V} \sum_{(f)} \frac{A_{f}^{2}}{\nu_{f}} \int \mathrm{d} x^{\prime} e^{\mathrm{i} f\left(x-x^{\prime}\right)} \Phi_{0}^{*}\left(x^{\prime}\right) \Phi_{0}(x) \\
& +\frac{1}{2 V} \sum_{(f)} \frac{A_{f}^{2}}{\nu_{f}} \int \mathrm{d} x \mathrm{~d} x^{\prime} e^{\mathrm{i} f\left(x-x^{\prime}\right)}\left|\Phi_{0}(x) \Phi_{0}\left(x^{\prime}\right)\right|^{2} .
\end{aligned}
$$

The subject of this work is to calculate the ground state energy $\mathcal{E}$ in the case of strong coupling for optical polaron when parameters $A_{f}$ and $\nu_{f}$ have the form $A_{f}=A /|f|, \nu_{f}=\nu$. We notice that in some works $[15,16]$

$$
\frac{H}{\omega}=-\Delta_{y}+\left(\frac{4 \pi \alpha}{\Omega}\right)^{1 / 2} \sum_{(q)} \frac{1}{q}\left(b_{q} e^{\mathrm{i} q x}+b_{q}^{+} e^{-\mathrm{i} q x}\right)+\sum_{(q)} b_{q}^{+} b_{q} .
$$

It's easy to see that the Hamiltonian (16) can be reduced to the form (21) if we change variables

$$
y=x / \zeta, \quad V=\Omega \zeta^{3}, \quad f=q / \zeta, \quad \zeta=\zeta_{0}=\frac{4 \pi \nu}{A^{2} m}
$$

and introduce notations

$$
\omega=\varepsilon^{2} \nu, \quad \alpha=\frac{A^{2}}{4 \pi \varepsilon \nu}\left(\frac{m}{2 \nu}\right)^{1 / 2}=\left(2 m \zeta_{0}^{2} \omega\right)^{1 / 2} .
$$

Performing in equation (20) summation over $f$

$$
\frac{1}{V} \sum(f) \frac{e^{\mathrm{i} f\left(x-x^{\prime}\right)}}{|f|^{2}}=\frac{1}{(2 \pi)^{3}} \int \mathrm{d} f \frac{e^{\mathrm{i} f\left(x-x^{\prime}\right)}}{|f|^{2}}=\frac{1}{4 \pi\left|x-x^{\prime}\right|}
$$

we have

$$
\Gamma=-\frac{\Delta_{x}}{2 m}-\frac{A^{2}}{4 \pi \nu} \int \mathrm{d} x^{\prime} \frac{\left|\Phi_{0}\left(x^{\prime}\right)\right|^{2}}{\left|x-x^{\prime}\right|}+\frac{A^{2}}{8 \pi \nu} \int \mathrm{d} x \mathrm{~d} x^{\prime} \frac{\left|\Phi_{0}(x) \Phi_{0}\left(x^{\prime}\right)\right|^{2}}{\left|x-x^{\prime}\right|} .
$$

Multiply equation (19 b) by $\Phi_{n}(x)$ and $\Phi_{0}(x)$ and integrate over $\mathrm{d} x$. So we can get for $n=0$

$$
-\frac{1}{2 m} \int \mathrm{d} x \Phi_{0}(x) \Delta_{x} \Phi_{0}(x)-\frac{A^{2}}{8 \pi \nu} \int \mathrm{d} x \mathrm{~d} x^{\prime} \frac{\left|\Phi_{0}(x) \Phi_{0}\left(x^{\prime}\right)\right|^{2}}{\left|x-x^{\prime}\right|}=E_{0} .
$$

Let's take scaling transformation with dimensionless parameter $\zeta$

$$
x \rightarrow y=\frac{x}{\zeta}, \quad \Phi_{n}(x) \rightarrow \Theta_{n}^{\zeta}(y)=\zeta^{3 / 2} \Phi_{n}(\zeta y) .
$$

Then

$$
\Gamma_{\zeta}=-\frac{\Delta_{y}}{2 m} \zeta^{-2}-\frac{A^{2}}{4 \pi \zeta} \int \mathrm{d} y^{\prime} \frac{\left|\Theta_{0}^{\zeta}\left(y^{\prime}\right)\right|^{2}}{\left|y-y^{\prime}\right|}+\frac{A^{2}}{8 \pi \nu \zeta} \int \mathrm{d} y \mathrm{~d} y^{\prime} \frac{\left|\Theta_{0}^{\zeta}(y) \Theta_{0}^{\zeta}\left(y^{\prime}\right)\right|^{2}}{\left|y-y^{\prime}\right|}
$$


where eigenfunctions $\Theta_{n}^{\zeta}$ satisfy the equation

$$
\Gamma_{\zeta} \Theta_{n}^{\zeta}(y)=E_{n} \Theta_{n}^{\zeta}(y)
$$

with normalization condition $\int \mathrm{d} y\left|\Theta_{n}^{\zeta}(y)\right|^{2}=1$. Multiply equation (25) by $\Theta_{n}^{\zeta}(y), \Theta_{0}^{\zeta}(y)$ and integrate over $y$ then put $n=0$. So we get $E_{0}=$ $\int \mathrm{d} y \Theta_{0}^{\zeta}(y) \Gamma_{\zeta} \Theta_{0}^{\zeta}(y)$. Starting from obvious equations

$$
\frac{\partial E_{0}}{\partial \zeta}=0, \quad \int \mathrm{d} y \Theta_{n}^{\zeta}(y) \frac{\partial}{\partial \zeta} \Theta_{n}^{\zeta}(y)=0
$$

we get

$$
\int \mathrm{d} y \Theta_{0}^{\zeta}(y)\left(\frac{\partial}{\partial \zeta} \Gamma_{\zeta}\right) \Theta_{0}^{\zeta}(y)=0
$$

and after differentiation we have

$$
\frac{1}{m \zeta^{3}} \int \mathrm{d} y \Theta_{0}^{\zeta}(y) \Delta_{y} \Theta_{0}^{\zeta}(y)+\frac{A^{2}}{8 \pi \nu \zeta^{2}} \int \mathrm{d} y \mathrm{~d} y^{\prime} \frac{\left|\Theta_{0}^{\zeta}(y) \Theta_{0}^{\zeta}\left(y^{\prime}\right)\right|^{2}}{\left|y-y^{\prime}\right|}=0 .
$$

From the other hand from equation (23) after transformation (24) it follows that

$$
-\frac{1}{2 m \zeta^{2}} \int \mathrm{d} y \Theta_{0}^{\zeta}(y) \Delta_{y} \Theta_{0}^{\zeta}(y)-\frac{A^{2}}{8 \pi \nu \zeta} \int \mathrm{d} y \mathrm{~d} y^{\prime} \frac{\left|\Theta_{0}^{\zeta}(y) \Theta_{0}^{\zeta}\left(y^{\prime}\right)\right|^{2}}{\left|y-y^{\prime}\right|}=E_{0}
$$

and finally from equation (26) and equation (27) we find the integral

$$
\frac{A^{2}}{8 \pi \nu \zeta} \int \mathrm{d} y \mathrm{~d} y^{\prime} \frac{\left|\Theta_{0}^{\zeta}(y) \Theta_{0}^{\zeta}\left(y^{\prime}\right)\right|^{2}}{\left|y-y^{\prime}\right|}=-2 E_{0} .
$$

It is convenient to put the parameter $\zeta$ to be equal

$$
\zeta=\zeta_{0}=\frac{4 \pi \nu}{A^{2} m},\left.\quad \Theta_{n}^{\zeta}(y)\right|_{\zeta=\zeta_{0}}=\Psi_{n}(y)
$$

and introduce a dimensionless one

$$
\alpha=\frac{A^{2}}{4 \pi \varepsilon \nu}\left(\frac{m}{2 \nu}\right)^{1 / 2}=\left(\varepsilon^{2} \nu 2 m \zeta_{0}^{2}\right)^{-1 / 2} .
$$

Then from equation (25) for $n=0$ we have that $\Psi_{0}(y)$ is an eigenfunction of equation

$$
\left\{-\Delta_{y}-2 \int \mathrm{d} y^{\prime} \frac{\left|\Psi\left(y^{\prime}\right)\right|^{2}}{\left|y-y^{\prime}\right|}-\lambda\right\} \Psi(y)=0
$$

corresponding to eigenvalue $\lambda_{0}$ which is equal to

$$
\lambda_{0}=6 m \zeta_{0}^{2} E_{0}=3 \frac{E_{0}}{\varepsilon^{2} \nu} \alpha^{-2} .
$$

It is the minimum eigenvalue of equation (30). For $n>0$ from equation (30)

$$
\left\{-\Delta_{y}-2 \int \mathrm{d} y^{\prime} \frac{\left|\Psi_{0}\left(y^{\prime}\right)\right|^{2}}{\left|y-y^{\prime}\right|}-\lambda_{0}+\alpha^{-2}\right\} \Psi_{n}(y)=\lambda_{n} \Psi_{n}(y),
$$


where an eigenvalue $\lambda_{n}$ is connected with $E_{n}$ by the expression

$$
\lambda_{n}=2 m \zeta_{0}^{2}\left(E_{n}-E_{0}\right)+\alpha^{-2}, \quad n>0 .
$$

Then let's apply successively equations (20), (22), (24), (28), (29), (33) to equation (19a). After transformations we can get

$$
\Delta_{2}=-\nu I(\alpha),
$$

where

$$
I(\alpha)=\int \mathrm{d} y \mathrm{~d} y^{\prime} \frac{Q\left(y, y^{\prime}\right)}{\left|y-y^{\prime}\right|} \Psi_{0}(y) \Psi_{0}^{*}\left(y^{\prime}\right)
$$

and

$$
Q\left(y, y^{\prime}\right)=\sum_{n>0} \frac{\Psi_{n}(y) \Psi_{n}^{*}\left(y^{\prime}\right)}{\lambda_{n}}
$$

So according to equation (29) $\alpha^{-1}=O(\varepsilon)$ together with equations (31), (34) we have

$$
\mathcal{E}=\varepsilon^{2} \nu\left\{\frac{\lambda_{0}}{3} \alpha^{2}-I(\alpha)+O\left(\alpha^{-2}\right)\right\} .
$$

Thus equations (30), (32), (34), (35) form closed way which permits us to calculate the second order approximation to the energy of optical polaron in the limit of strong coupling.

Then we can simplify the problem by introducing the spherical coordinates $y=(r, \Theta, \varphi)$ and eliminating angle variables. So $\Psi_{0}(y)$ becomes a spherically symmetric function $\Psi_{0}(y)=\Psi_{0}(r)$. So we can fulfil integration over angle variables $\Theta, \varphi$ in the left-hand side of equation (30) and equation (32). Thus

$$
\int \mathrm{d} y^{\prime} \frac{\left|\Psi\left(y^{\prime}\right)\right|^{2}}{\left|y-y^{\prime}\right|}=\frac{q(r)}{r},
$$

where

$$
q(r)=1-4 \pi \int_{r}^{\infty} \mathrm{d} r^{\prime}\left(1-\frac{r}{r^{\prime}}\right) r^{\prime 2} \Psi^{2}\left(r^{\prime}\right) .
$$

It's easy to see that $q(r)$ is a monotonically increasing function with domain $[0,1)$ :

$$
q(O)=0, \quad \lim _{r \rightarrow \infty} q(r)=1 .
$$

Let's introduce the function $\rho(r)=2 \sqrt{\pi} r \Psi(r)$, satisfying the boundary and normalization conditions

$$
\rho(o)=0 ; \quad \lim _{r \rightarrow \infty} \rho(r)=0, \quad \int_{0}^{\infty} \mathrm{d} r \rho^{2}(r)=1 .
$$

In accordance with equations (30), (32), (38) the function $\rho(r)$ matches the system of equations

$$
\left\{\begin{array}{l}
\rho^{\prime \prime}(r)+\frac{2}{r} q(r) \rho(r)=-\lambda \rho(r) \\
q^{\prime \prime}(r)=-\rho^{2}(r) / r
\end{array}\right.
$$


with boundary conditions (39), (40). This system can be resolved numerically by the method of shooting from points $r=0, r=\infty$. The eigenvalue $\lambda_{0}$ is determined from smoothness condition for function $\rho_{0}(r)$ which has no pole in the interval $(0, \infty)$

$$
\lambda_{0}=-0.3 \ldots
$$

To exclude angle variables from equations (35), (36) let's single out explicitly eigenvalues of the angle part of the operator $\Delta_{y}$

$$
Q\left(y, y^{\prime}\right)=\sum_{\substack{n, l, m \\(n>0)}} \frac{\Psi_{n, l, m}(y) \Psi_{n, l, m}\left(y^{\prime}\right)}{\lambda_{n, l, m}}
$$

where $\Psi_{n, l, m}(y)$ and $\lambda_{n, l, m}$ are eigenfunctions and eigenvalues of the problem

$$
\left[-\Delta_{y}-2 \frac{q(r)}{r}-\lambda_{0}+\alpha^{-2}\right] \Psi_{n, l, m}(y)=\lambda_{n, l, m} \Psi_{n, l, m}(y) .
$$

It is clear that the eigenfunction $\Psi_{000}(r)$ with the smallest eigenvalue $\lambda_{000}$ is $\Psi_{000}(r)=\Psi_{0}(r), \lambda_{000}=\alpha^{-2}$. Summation in equation (43) is fulfilled over all $(n, l, m) \neq(0,0,0)$. Let's decompose functions $\Psi_{n, l, m}(y)$ using spherical harmonics:

$$
\Psi_{n, l, m}(y)=\frac{\rho_{n, l}(r)}{r} Y_{l, m}(\Theta, \varphi)
$$

taking into account the normalization condition $\int_{0}^{\infty} \mathrm{d} r\left(\rho_{n, l}(r)\right)^{2}=1$. Then the equation (44) can be reduced to

$$
\left[-\frac{\partial^{2}}{\partial r^{2}}-2 \frac{q(r)}{r}+\frac{l(l+1)}{r^{2}}-\lambda_{0}+\alpha^{-2}\right] \rho_{n, l}(r)=\lambda_{n, l} \rho_{n, l}(r) .
$$

Let's introduce the function

$$
G_{l}\left(r, r^{\prime}\right)=\sum_{n>0}\left(\lambda_{n, l}\right)^{-1} \rho_{n, l}(r) \rho_{n, l}\left(r^{\prime}\right) .
$$

According to equation (45) it satisfies the equation

$$
\begin{aligned}
\hat{\mathscr{L}}_{r} G_{l}\left(r, r^{\prime}\right) & =\delta\left(r-r^{\prime}\right)-\delta_{l, 0} \rho_{0}(r) \rho_{0}\left(r^{\prime}\right), \\
\hat{\mathscr{L}}_{r} & =-\frac{\partial^{2}}{\partial r^{2}}-2 \frac{q(r)}{r}+\frac{l(l+1)}{r^{2}}-\lambda_{0}+\alpha^{-2},
\end{aligned}
$$

where $\rho_{0}(r)$ is the solution to the system (41) with $\lambda_{0}$ given by equation (42). Because the eigenfunctions $\rho_{n, l}(r)$ comply with boundary conditions

$$
\rho_{n, l}(0)=0, \quad \lim _{r \rightarrow \infty} \rho_{n, l}(r)=0,
$$

so those for the functions $G_{l}\left(r, r^{\prime}\right)$ are:

$$
G_{l}\left(0, r^{\prime}\right)=G_{l}\left(\infty, r^{\prime}\right)=G_{l}(r, 0)=G_{l}(r, \infty)=0 .
$$


Let $g_{l}^{(1)}(r)$ and $g_{l}^{(2)}(r)$ be solutions to the uniform equations with boundary conditions:

$$
\left\{\begin{array} { l } 
{ \hat { \mathscr { L } } _ { r } g _ { l } ^ { ( 1 ) } ( r ) = 0 , } \\
{ g _ { l } ^ { ( 1 ) } ( 0 ) = 0 , }
\end{array} \quad \left\{\begin{array}{l}
\hat{\mathscr{L}}_{r} g_{l}^{(2)}(r)=0 \\
g_{l}^{(2)}(\infty)=0
\end{array}\right.\right.
$$

Then $G_{l}\left(r, r^{\prime}\right)$ can be represented as

$$
\begin{aligned}
G_{l}\left(r, r^{\prime}\right)= & W^{-1}\left\{g_{l}^{(1)}(r) g_{l}^{(2)}\left(r^{\prime}\right) \sigma\left(r-r^{\prime}\right)+\right. \\
& \left.g_{l}^{(1)}\left(r^{\prime}\right) g_{l}^{(2)}(r) \sigma\left(r-r^{\prime}\right)\right\}-\alpha^{2} \delta_{l, 0} \rho_{0}(r) \rho_{0}\left(r^{\prime}\right),
\end{aligned}
$$

where $W$ is the Wronskian

$$
W=g_{l}^{(1)}(r) \frac{\partial}{\partial r} g_{l}^{(2)}(r)-g_{l}^{(2)}(r) \frac{\partial}{\partial r} g_{l}^{(1)}(r)=\mathrm{const},
$$

and $\sigma(r)$ is a step-function $\sigma(r)=\left\{\begin{array}{ll}1, & r>0, \\ 0.5, & r=0, \\ 0, & r<0 .\end{array}\right.$ In this case we have for the integral (35)

$$
I(\alpha)=\sum_{l} I_{l}(\alpha), \quad I_{l}(\alpha)=\int_{0}^{\infty} \int_{0}^{\infty} \mathrm{d} r \mathrm{~d} r^{\prime} G_{l}\left(r, r^{\prime}\right) \rho_{0}(r) \rho_{0}\left(r^{\prime}\right) H_{l}\left(r, r^{\prime}\right),
$$

where

$$
H_{l}\left(r, r^{\prime}\right)=\iint \mathrm{d} \Omega \mathrm{d} \Omega^{\prime} \sum_{m} Y_{l, m}(\Theta, \varphi) Y_{l, m}\left(\Theta^{\prime}, \varphi^{\prime}\right) \frac{1}{4 \pi\left|x-x^{\prime}\right|} .
$$

Taking into account properties of spherical harmonics we get

$$
H_{l}\left(r, r^{\prime}\right)=\frac{r^{l}}{\left(r^{\prime}\right)^{l+1}} \sigma\left(r^{\prime}-r\right)+\frac{\left(r^{\prime}\right)^{l}}{r^{l+1}} \sigma\left(r-r^{\prime}\right) .
$$

It's obvious that $I_{l}(\alpha)=O\left(\alpha^{0}\right)$, so it follows from equation (37) that

$$
\mathcal{E}=\varepsilon^{2} \nu\left\{\frac{\lambda_{0}}{3} \alpha^{2}+B+O\left(\alpha^{-2}\right)\right\}
$$

where

$$
B=-\lim _{\alpha \rightarrow \infty} I(\alpha)=-\lim _{\alpha \rightarrow \infty} I_{0}(\alpha)-\lim _{\alpha \rightarrow \infty} \sum_{l=1}^{\infty} I_{l}(\alpha)
$$

and moreover to find $I_{l}$ for $l>0$ it's possible to put $\alpha^{-2}=0$ in equation (46). The system of equations (32) has been solved numerically for $l=0, \ldots, 4$ :

$$
\begin{aligned}
& I_{0}=0.174, \quad I_{1}=0.86674, \quad I_{3}=0.16412, \\
& I_{2}=0.31551, \quad I_{4}=0.10053 \text {. }
\end{aligned}
$$

And magnitudes of $I_{l}(\infty)$ were derived on the basis of these calculated values by means of the following extrapolation law:

$$
I_{l}(\infty)=\frac{c}{l^{2}}+\frac{d}{l^{3}}+\frac{e}{l^{4}}+\cdots .
$$

As a result the following value for the $B$ parameter was derived: $B=$ $-2.0 \ldots$, which differs from the result of the work $[16]: B=-2.836 \ldots$ 


\title{
References
}

[1] Balalaev N.K., Lakhno V.D. Self-consistent solutions in the continuous $F$ center model (Preprint ONTINSBT, Acad. Nauk USSR).

[2] Lakhno V.D. Excited polaron states in condensed medium. Manchester University Press.

[3] Achiezer A.I., Pomeranchuk I.B. // JETP, 1946, vol. 16, p. 391 (in Russian).

[4] Tiablikov S.V. // JETP, 1948, vol. 18, p. 1023 (in Russian).

[5] Tiablikov S.V. // Doklady of Ukrainian Academy, 1950, vol. 6, p. 3 (in Russian).

[6] Pekar S.I. // JETP, 1949, vol. 19, p. 769 (in Russian).

[7] Pekar S.I. Studies in the electronic theory of crystal. Moscow, Gostekhizdat, 1951 (in Russian).

[8] Bogolubov N.N. (Jr.), Fam Le Kien, Shumovsky A.S. // Teor. Mat. Fiz., 1982, vol. 52, p. 423 (in Russian).

[9] Bogolubov N.N. (Jr.), Fam Le Kien, Shumovsky A.S. // Physica, 1984, vol. 128 A, p. 82.

[10] Bogolubov N.N. (Jr.), Fam Le Kien, Shumovsky A.S. // Physica, 1985, vol. 133 A, p. 413.

[11] Born M. "Oppenhiemer". // Ann. der Physik, 1927, vol. 84, p. 457.

[12] Bogolubov N.N. A new form of adiabatic perturbation theory in the problem of particle coupling with a quantum field. // Ukr. Mat. Zhurn., 1950, vol. 2, No 2, 3 (in Russian).

[13] Lakhno V.D., Chuev G.N. // Fiz. Tverd. Tela, 1989, vol. 31, No 2, p. 23.

[14] Bogolubov N.N. Preprint JINR E-2-90-535, 1990, see also: Proceedings of the Italian-Soviet Workshop: Advances in Theoretical Physics, 23-28 October, 1990, Dubna, JINR (World Scientific, ed. by E.R.Cafaniello).

[15] Krivoglaz M.A., Pekar S.I. The trace method for the conductivity electrons. // Izvest. Akad. Nauk USSR, Ser. Phys., 1957, vol. 21, No 1, p. 1632.

[16] Miyake S.J. // J. Phys. Soc. of Japan, 1976, vol. 41, No 3.

\section{НАБЛИЖЕННЯ ДРУГОГО ПОРЯДКУ ДЛЯ ОПТИЧНОГО ПОЛЯРОНУ У ВИПАДКУ СИЛЬНОГО ЗВ'ЯЗКУ}

\author{
М.М.Боголюбов (мол.)
}

Пропонується метод побудови наближення другого порядку для основного стану енергії класу модельних гамільтоніанів з лінійним типом взаємодії по бозівських операторах у випадку сильного зв'язку. Для застосування зазначеного методу розглядається модель полярону й пропонується набір нелінійних диференціальних рівнянь для розрахунку основного стану енергії у випадку сильного зв'язку. Також розглядається радіально симетричний випадок. 